\title{
Experimental validation of bidirectional reflection and transmission distribution measurements of specular and scattering materials
}

\author{
Lars O. Grobe and Stephen Wittkopf ${ }^{a}$; \\ Peter Apian-Bennewitz ${ }^{b}$; \\ Jacob C. Jonsson and Mike Rubin ${ }^{c}$ \\ ${ }^{a}$ Solar Energy Research Institute of Singapore, Singapore; \\ ${ }^{b}$ PAB Advanced Technologies Ltd, Freiburg, Germany; \\ ${ }^{c}$ Environmental Energy Technology Division, Lawrence Berkeley National Laboratory, \\ Berkeley, California, USA
}

\begin{abstract}
The development of advanced materials for facades aims to achieve higher energy efficiency of buildings. Successful application of these materials depends on the availability of reliable characterization data. While data derived from integrated measurements of transmission and reflection is widely available, it does not allow to characterize the angular dependence of the performance of such materials. The Bidirectional Reflection-Transmission Distribution (BRTD) can be measured by commercially available Gonio-Photometers and, complimenting integrated transmittance and reflectance, allows the assessment of facade materials and thus supports both their development and application. Validation of the obtained data is crucial to back these measurements.

Integration of validation procedures into the operation of a characterization laboratory allowing a well-defined approach to quality control is presented for a range of typical material and sample types:

- consistency checks of measurement data

- cross-checking of integrated material properties derived from BRTD data with integrating sphere measurements

- round-robin comparison between laboratories using comparable devices

The results of of these first measurements are discussed. Potential to further improve the availability of reliable angular resolved characterization data for the building sector is identified.
\end{abstract}

Keywords: bidirectional reflection transmission distribution, goniophotometer, validation

\section{Introduction}

The application, optimization and assessment of directional selective and redirecting structures used in advanced daylighting require angular resolved characterization of reflection and transmission. The exitance distribution depending on the incident angle can be measured using Goniophotometers. Several approaches have been proposed to design devices to perform such measurements, which can be categorized as scanning and image-based. The resulting set of reflection and transmission readings for each ingoing and outgoing direction is described as the Bidirectional Reflection and Transmission Distribution (BRTD). ${ }^{1}$ Interpolation and application of models

Further author information:

Solar Energy Research Institute of Singapore, 7 Engineering Drive 1, Block E3A, \#06-01, Singapore 117574;

PAB Advanced Technologies Ltd, D-79114 Freiburg, Germany;

Environmental Energy Technology Division, Lawrence Berkeley National Laboratory, 1 Cyclotron Road, Berkeley, 94720 California, USA

Lars O. Grobe, Stephen Wittkopf, Peter Apian-Bennewitz, Jacob C. Jonsson and Mike Rubin, "Experimental validation of bidirectional reflection and transmission distribution measurements of specular and scattering materials", Photonics for Solar Energy Systems III, Ralf B. Wehrspohn; Andreas Gombert, Editors, Proc. SPIE 7725, 772510 (2010). https://doi.org/10.1117/12.854011

Copyright 2010 Society of Photo Optical Instrumentation Engineers. One print or electronic copy may be made for personal use only. Systematic reproduction and distribution, duplication of any material in this paper for a fee or for commercial purposes, or modification of the content of the paper are prohibited. 
allow to derive a function to describe the distribution at any incident and outgoing direction. This Bidirectional Reflection and Transmission Distribution Function (BRTDF) depends at least on the incoming and outgoing direction $\left(\vec{\chi}_{\text {in }}, \vec{\chi}_{\text {out }}\right)$ :

$$
L_{\text {out }}\left(\vec{\chi}_{\text {out }}\right)=\int_{\vec{\chi}_{\text {in }}}^{\Omega_{\text {in }}=4 \pi} \operatorname{BRTDF}\left(\vec{\chi}_{\text {out }}, \vec{\chi}_{\text {in }}\right) \cdot L_{i n}\left(\vec{\chi}_{\text {in }}\right) \cdot \cos \left(\alpha_{i n}\right) \cdot d \Omega_{\text {in }}
$$

With $L_{\text {out }}\left(\vec{\chi}_{\text {out }}\right)$ being the radiance scattered into direction $\vec{\chi}_{\text {out }}, L_{\text {in }}\left(\vec{\chi}_{\text {in }}\right)$ the incident light distribution and $\alpha_{i n}$ the angle between the surface normal and the light source direction $\vec{\chi}_{i n}$. For a light source assumed to emit parallel light leading to an incident irradiance $E_{i n}$, this is commonly simplified into:

$$
\operatorname{BRTDF}\left(\vec{\chi}_{\text {out }}, \vec{\chi}_{\text {in }}\right)=\frac{L_{\text {out }}\left(\vec{\chi}_{\text {out }}\right)}{E_{\text {in }}}
$$

Since Torrance and Sparrow ${ }^{2}$ introduced the theoretical foundation for the development of BRTDFs, the improvements in the design of Gonio-Photometers as well as the increase in availability of computing resources as a requirement to apply such data to models for simulation have led to a refinement of measurements. A view on the publicly available databases for measurement data gives evidence - the range starts at 205 combinations of incident and outgoing directions per sample for the "Columbia-Utrecht Reflectance and Texture Database ${ }^{\text {(3 }}$ to image based measurement databases such as Matusik's ${ }^{4}$ with more then $10^{6}$ directions per sample. Ongoing work such as spectral, spatially or time-resolved BRTD measurements lead to further increases. Validation studies mainly on the field of appearance have been presented including resampling of data-sets from different measurement devices. ${ }^{5}$ The continuing refinement of measurements, ${ }^{6}$ as well as the attempts to match the empirical data with theoretical models, leads to the need for control of the errors and uncertainties introduced both by measuring and fitting data, especially if the results are to be used for further optimization or to be included into libraries used to predict performance of materials and components in simulations. Three approaches to control the validity and identify error sources relevant in the characterization of building materials' performance as relevant for energy efficiency are proposed:

1. consistency checks on the results from measurements using one device

2. comparison of the results from measurements using a goniophotometer with integrating measurements

3. round-robin measurements leading to a comparison of results from similar devices

\section{INSTRUMENTATION USED}

All three laboratories participating in the study operate a commercially available scanning Goniophotometer of the same model line (PAB pgII-B1). ${ }^{7}$ A configurable lamp assembly points at a sample holder, which can be rotated in two axis varying the incident angles $\left(\phi_{i n}, \theta_{i n}\right)$. A detector head performs a continuous rotational movement around the sample center on different paths that cover a sphere. At chosen time intervals, the location of the detector head and the radiance detected are recorded. The recording time interval (typically $1 \mathrm{~ms}$ ) and the density of scan paths hereby define the resolution of the scan. Angular ranges of interest can be rescanned at a higher resolution, which is the default procedure for the outgoing directions of expected specular reflection and transmission. The result is a scattered point set of varying density on an imaginary spherical surface with associated luminance readings. At least two such measurements are performed. The first one scans the unobstructed beam after stabilization according to the lamp characteristics. This beam is referred to as the reference beam. Than one or more samples are mounted on the sample holder and scanned. Relating the reading with the sample mounted to the integral value of the unobstructed beam for any $\left(\phi_{\text {in }}, \theta_{\text {in }}\right),\left(\phi_{\text {out }}, \theta_{\text {out }}\right)$ leads to the reflection and transmission of the sample dependent on incoming and outgoing directions. Interpolation or application of suitable reflection and transmission models results in a complete description of the Bidirectional Reflection / Transmission Distribution Function (BRTDF) of the sample. 
The configurations of the instruments vary in such as they offer different levels of automatization on the sample holder, as well as in the details of the lamps and filter-detector combinations used. The critical components, such as lamps, filters and detectors, are listed. While each system offers more then the described configurations, a common setup was agreed upon including a halogen lamp, a Si-photocell and a hot mirror cutting off the near infrared range from $700 \mathrm{~nm}$ to $1100 \mathrm{~nm}$. The filter compensates the fact that both the lamp's emission spectra and the sensor's response have their maxima in the near infrared at about 900nm, and an unfiltered measurement would be dominated by the sample characteristics in the near IR range.

\section{SAMPLE SET}

A set of samples was made available to each of the laboratories. The samples were chosen to cover a wide range of properties in reflection, transmission and scatter. The samples shared are not certified references, but reflect products as commercially available. However, this introduces the uncertainty of variance between supposedly

\begin{tabular}{|c|c|c|c|}
\hline & LBNL & PAB & SERIS \\
\hline \hline lamp & Osram Halostar 50W & Osram Halostar 50W & Osram Halostar ES 35W \\
\hline detector & $\mathrm{Si}$ & $\mathrm{Si}$ & $\mathrm{Si}$ \\
\hline filters & $\begin{array}{c}\text { NIR-filter 900-1100nm } \\
\text { attached to detector }\end{array}$ & $\begin{array}{c}\text { NIR-filter 900-1100nm on } \\
\text { lamp assembly }\end{array}$ & $\begin{array}{c}\text { NIR-filter 900-1100nm on } \\
\text { lamp assembly }\end{array}$ \\
\hline sample holder & $\begin{array}{c}\text { semi-automatic } \\
\left(\text { manual } \phi_{i n}\right)\end{array}$ & $\begin{array}{c}\text { automatic } \\
\left.\text { (both } \phi_{\text {in }}, \theta_{i n}\right)\end{array}$ & $\begin{array}{c}\text { semi-automatic } \\
\left.\text { (manual } \phi_{i n}\right)\end{array}$ \\
\hline
\end{tabular}

Table 1. Configurations used for the round-robin tests at the three participating laboratories.

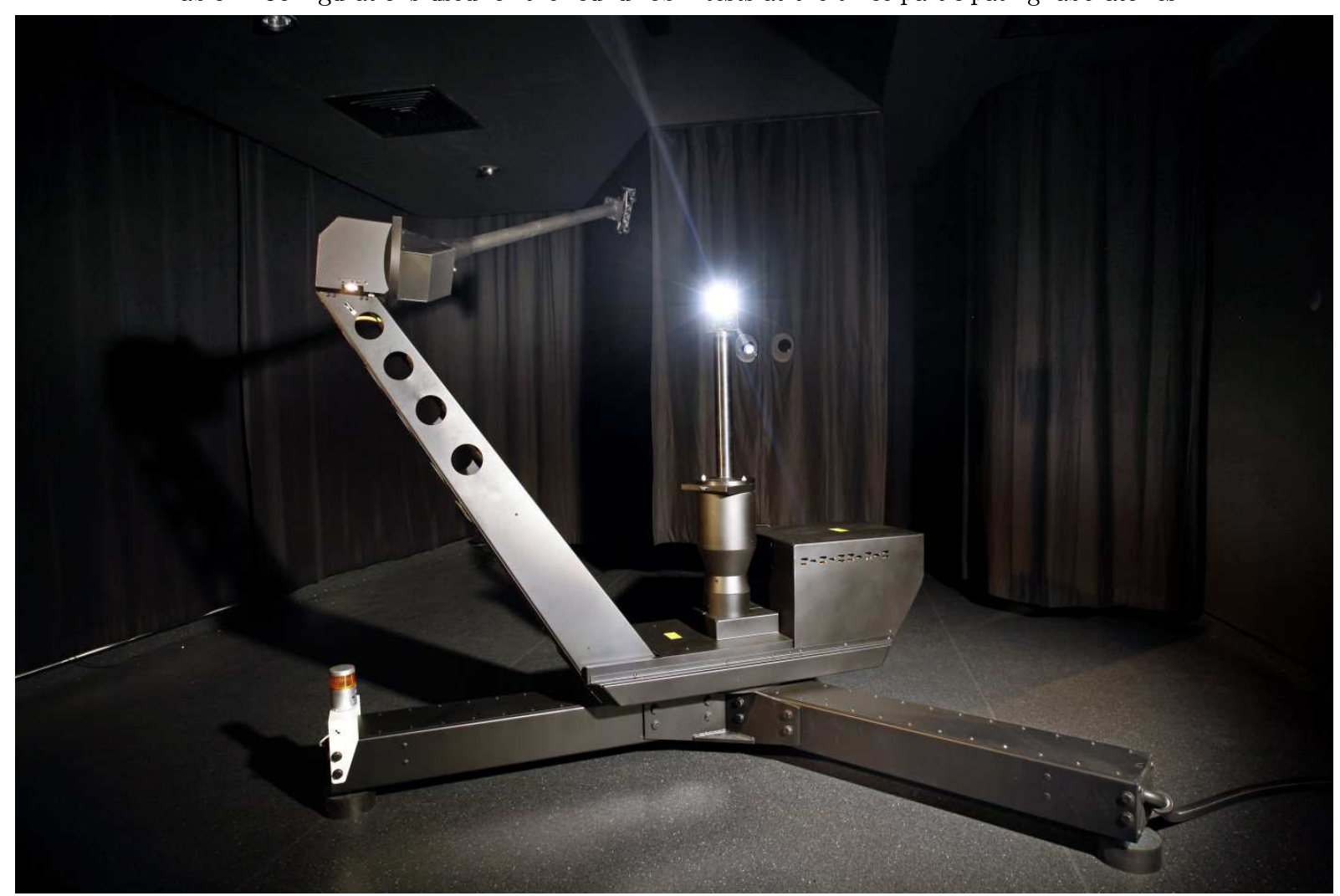

Figure 1. The goniophotometer as in operation at SERIS. The sample holder is shown in the center and the sample hit by light emitted from a source that is located behind a curtain to minimize straylight. 


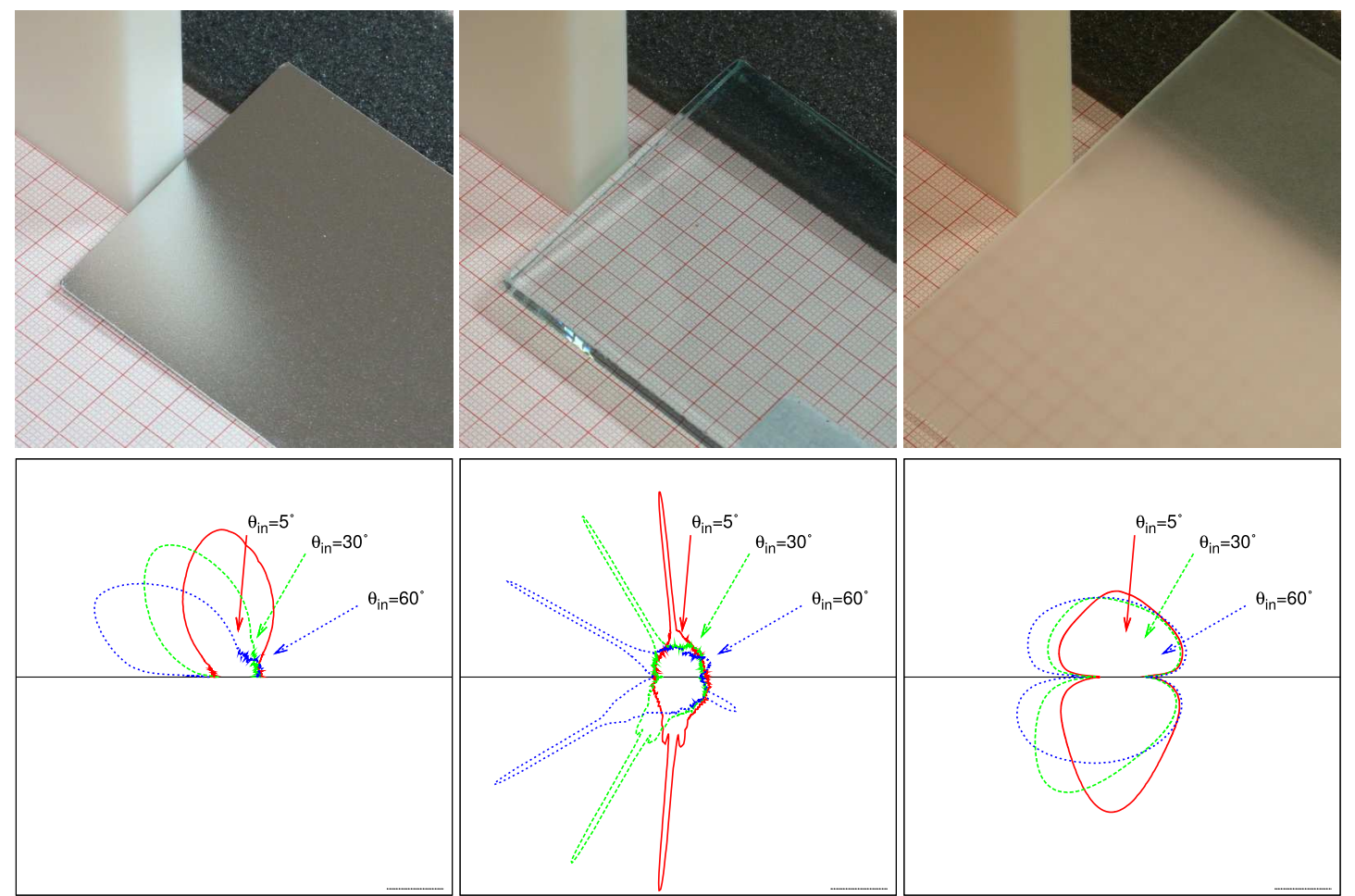

Table 2. Photographs and BRTD profiles in the scatter plane $\left(\phi_{\text {out }}=0\right)$ for three samples used for the round-robin tests at the three participating laboratories (left to right): scattering aluminum front-side mirror miro20-2000, clear low-e glass clear1, scattering ground glass edm45656. The profiles are shown in a logarithmic scale and cosine-weighted.

identical samples. The properties of the samples are not known in advance besides publicly available information from vendors' data-sheets.

\section{INSTRUMENT-BASED CONSISTENCY CHECKS}

To identify any internal, instrument-related influences on the measurement as well as external influences due to environmental conditions, the data acquired from the device was assessed at different levels of the measurement process. It is important to note that, as the measurement itself is relative to a reference measurement that is performed at the beginning (and preferably at the end) of any measurement series, the device does not require a quantitative calibration. Still, the optical, mechanical and electrical characteristics of the device and its environment are assumed to be stable during the measurement session. Any deviation from this assumption would introduce error into the measurement.

\subsection{Beam stability}

According to previous experiences with halogen lamps, a stabilization time of 30 minutes was assumed. Changes in the beam flux between the initial reference beam and the following measurements systematically distort the result. Typically a series of measurements would be performed with increasing incident angles, and a continuous fall-off in the beam intensity thus would lead to a systematic under-estimation of higher incident angles. To verify the stability of the beam flux, a repeated measurement of the unobstructed beam over an angle of 6 degree from the optical axis was performed, starting immediately after switching on the lamp. As expected, during the first 30 minutes, a significant fall-off of the beam flux was observed. However, the stabilization occurred later then expected, probably due to a change in the lamp configuration. Thus for all following measurements a stabilization time of 45 minutes is accounted for. The time interval between reference beam measurement 


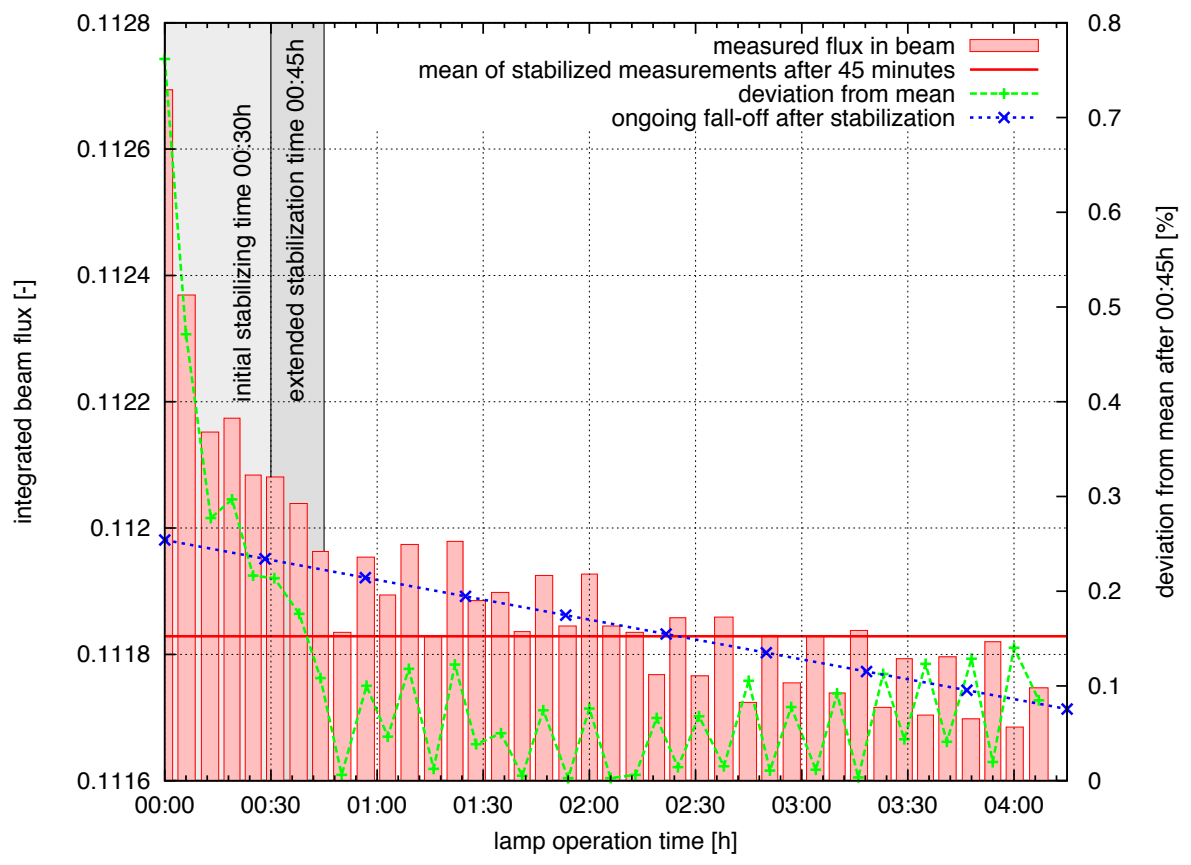

Figure 2. Stabilization of beam flux after power-on of the halogen lamp. Indicated by the gray background is the initially assumed stabilization time of 30 minutes, that was increased to 45 due to this study. After that time, an ongoing fall-off of $0.06 \%$ per hour was determined.

together with the ongoing decrease of beam-flux result in a systematic measurement error, which can be mostly controlled by the measurement routine.

The optical bench carrying the lamp assembly allows modifications of the beam characteristics. One important parameter that is typically adjusted according to requirements is the beam diameter. For samples with coarsely grained structures, the ability to increase the beam diameter to effectively cover a representative range of the surface is in fact more critical then the resolution. A measurement with a defocused beam leads to averaging over a larger angle and thus shows a different result, but is still valid. However the decreasing intensity related to the beam diameter leads to a decrease in the signal. The flexibility of the optical bench however implies the need of consistency checks and regular measurement of the reference beam. We show the beam characteristics such as the alignment of the beam in the optical axis and the beam angle for one given measurement.

\subsection{Detector response}

Assuming a stable beam flux and sample properties, the response of the detector system is critical for the validity of the measurement. To cover the wide range of expected irradiance readings especially for highly specular samples, the detector signal is fed into an adaptive amplifier system before the conversion of the signal into a digital value. Both the switching between the amplifier levels and the response of the detector when exposed to quickly changing irradiance levels have to be fast enough to match the data frequency of $1 \mathrm{~ms}$.

\subsection{Site conditions}

Measurements are performed relative to a reference measurement, that is supposed to include all the same environmental influences as the subsequent measurements. This makes the process rather insensitive towards 
external factors such as minor constant light leakages or smaller sources in the room. Sources can however affect a measurement when they are not covered by the reference beam measurement, e.g. reflection from a sample to a reflective wall surface. For specular samples, this effect can lead to significant measurement error, if specular reflective surfaces are present in the scatter plane. Efforts were taken to reduce such reflections by covering most of the walls with black curtains. For visible light, the curtain's fabric shows a reflectance below $5 \%$, which however increases to about $30 \%$ in the infrared range. The reflectance of ceiling and floor are considered to be less significant for this specular two-bounce reflection, as they are above or below the scattering plane. At SERIS, the wall facing the reflecting side of a sample however is painted black and not covered by a curtain during the measurements.

\section{INTEGRATED TRANSMITTANCE / REFLECTANCE RESULTS}

By integrating the transmission and reflection for all angles of the hemispheres on both sides of the sample, the direct-hemispherical transmittance and reflectance can be derived for a given incident angle. These can be directly compared as long as both all the emission spectra of the lamps used are identical and the detector used have the same spectral response. We compare the integrals of the measurements for each sample and each incident angle $\left(\theta_{\text {in }}=5,15,30,45,60\right.$ degrees) as measured at each participating laboratory.

The spectral response of the chosen configuration for the goniophotometer is calculated from the spectral response of the photocell, the transmission of the hot-mirror and a synthetic black-body radiation spectrum at $3,000 \mathrm{~K}$ as assumed for the lamp. The resulting spectrum is normalized to a mean of 1 and multiplied by the reflection and transmission spectra of the samples as measured on using an integrating sphere. The integrals of these response-corrected spectra are compared to the integrals of three BRTD measurements done at SERIS using the Si-detector, at 8 degrees for reflectance and 0 degrees for transmittance.

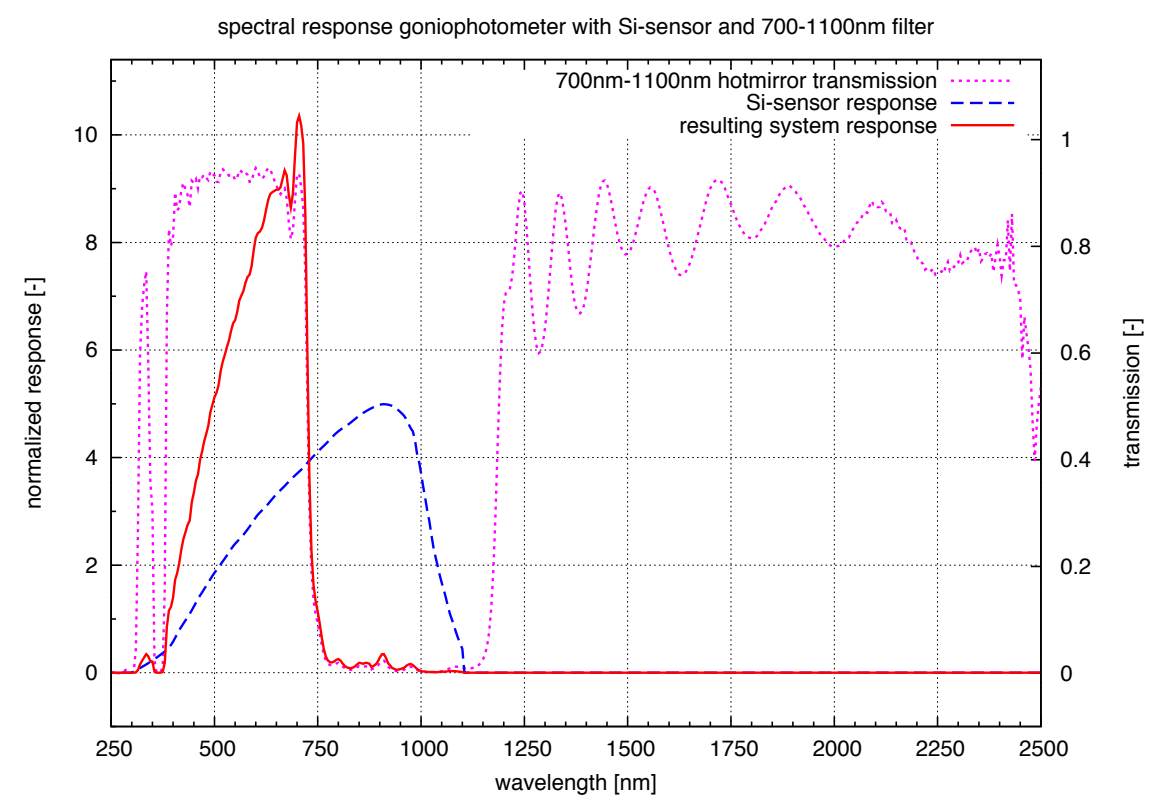

Figure 3. Spectral response of the goniophotometer with Si-detector and hot-mirror blocking wavelengths in the range of 700-1100nm. The resulting response curve was applied to the reflection and transmission spectra measured using an integrating sphere to allow direct comparison with the integrated BRTD measurements. 


\begin{tabular}{|c|c|c|c|c|}
\hline \multirow[b]{2}{*}{ sample ID } & \multicolumn{2}{|c|}{ integrating sphere } & \multicolumn{2}{|c|}{ goniophotometer } \\
\hline & $\begin{array}{c}\text { transmission } \\
\text { corrected } \\
T\end{array}$ & $\begin{array}{c}\text { reflectance } \\
\text { corrected } \\
R\end{array}$ & $\begin{array}{c}\text { transmission } \\
T\end{array}$ & $\begin{array}{c}\text { reflectance } \\
R \\
\end{array}$ \\
\hline clear1 & 0.6979 & 0.0801 & 0.7003 & 0.0777 \\
\hline edm45656 & 0.7818 & 0.1413 & 0.8118 & 0.1440 \\
\hline miro20-2000 & - & 0.9038 & - & 0.9199 \\
\hline
\end{tabular}

Table 3. Results from integrated transmission ( at 0 degrees) and reflectance (at 8 degrees) measurements using a 150mm integrating sphere with a Perkin Elmer Lambda 9500 spectrometer compared to integration of BRTD measurement for Si-sensor and 700nm-1100nm hot mirror. The transmission and reflectance spectra were corrected by applying the filter transmission spectrum and the spectral response of the sensor (transmission corrected).

Furthermore, the samples are measured using a second detector configuration at each laboratory, equipped with a $\mathrm{v}(\lambda)$-filter, at 8 degrees for the reflectance, 0 degrees for the transmittance measurement. The $\mathrm{v}(\lambda)$-filter shows a transmittance that, together with the response of the detector it is applied to, results in a spectral response close to the CIE standard observer. ${ }^{8}$ The results can thus be compared to the transmittance and reflectance for visible light as measured on a spectrometer with integrating sphere, where the spectral adoption to the CIE observer is implemented by a software algorithm.

The standard measurement procedure, which is refining the region of the expected peak and performs a rather coarse scan on the remaining directions thus cannot capture all of these regions. This leads to a systematic deviation in the interpolation, which is clearly visible on a polar surface plot based on the measurement locations for a clear aluminum mirror showing anisotropic reflection. The interpolation does not make any assumptions on the surface characteristics by applying reflection models, but connects locations to generate a net of Delaunay-triangles. Regions showing slopes outside the expected beam tend to get underestimated, which becomes especially significant for samples with more then one reflective and transmissive peak, e.g. in the case of retro-reflection. To get a better understanding of the systematic underestimate, a modified scan path is applied, leading to a refinement over not only the expected peak region (opposite the incident direction) but refining the full sphere measurement. Comparing the two measurements shows however shows the little impact of the regions outside the peak measurement and affected by the anisotropy on the BRTD's integral in this case. However the interpolation error for high variance beyond the expected peak region has to be considered and can be answered either by adapting the resolution of the scan or by fitting material models in the interpolation instead of a triangulation algorithm.

\begin{tabular}{|c|c|c|c|c|c|}
\hline sample & LBNL & PAB & SERIS & $\begin{array}{c}\text { mean } \\
\text { (from } \\
\text { BRTD) }\end{array}$ & $\begin{array}{c}\text { integrating } \\
\text { sphere }\end{array}$ \\
\hline miro20-2000 & 0.9164 & 0.9621 & 0.9150 & 0.9312 & 0.9368 \\
\hline clear1 & 0.0801 & 0.0882 & 0.0783 & 0.0822 & 0.0933 \\
\hline edm45656 & 0.1527 & 0.1590 & 0.1366 & 0.1494 & 0.1499 \\
\hline
\end{tabular}

Table 4. Results from integrated reflection measurements at 8 degrees using $\mathrm{v}(\lambda)$ filter on Goniophotometers at all three participating labs compared to integrating sphere measurement.

\begin{tabular}{|c|c|c|c|c|c|}
\hline sample & LBNL & PAB & SERIS & $\begin{array}{c}\text { mean } \\
\text { (from } \\
\text { BRTD) }\end{array}$ & $\begin{array}{c}\text { integrating } \\
\text { sphere }\end{array}$ \\
\hline clear1 & 0.7257 & 0.7393 & 0.7154 & 0.7268 & 0.7292 \\
\hline edm45656 & 0.7958 & 0.8094 & 0.7932 & 0.7995 & 0.7856 \\
\hline
\end{tabular}

Table 5. Results from integrated transmission measurements at 0 degrees using $\mathrm{v}(\lambda)$ filter on Goniophotometers at all three participating labs compared to integrating sphere measurement. 


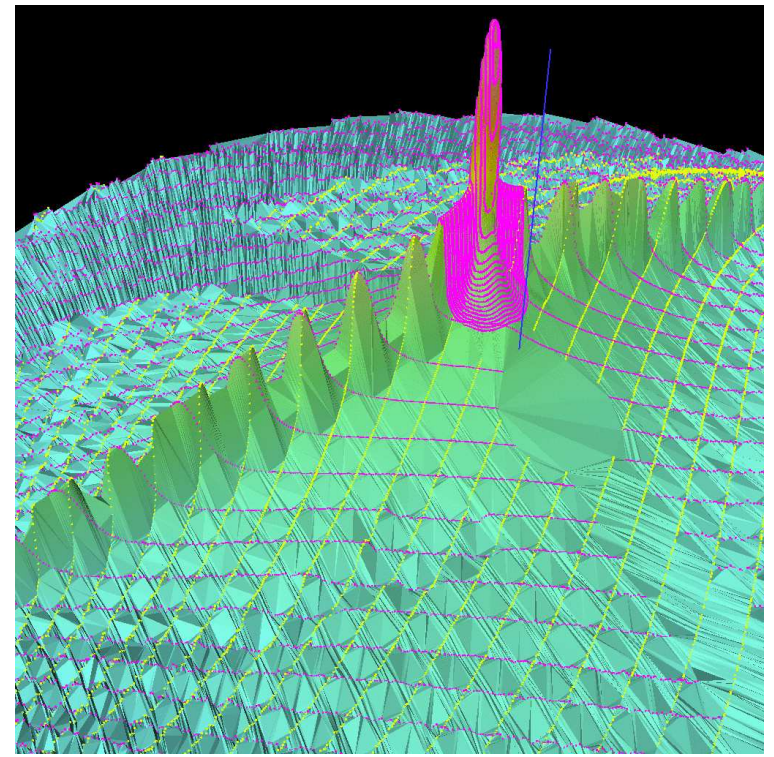

Figure 4. Reflection distribution on a clear aluminum front-side mirror at 8 degrees incident angle as derived. The interpolation between the measurement locations (marked as yellow and magenta dots) clearly cannot account for the steep slopes lying outside the refined beam region. A logarithmic scale has been applied to make the low reflection values outside the peak area visible.

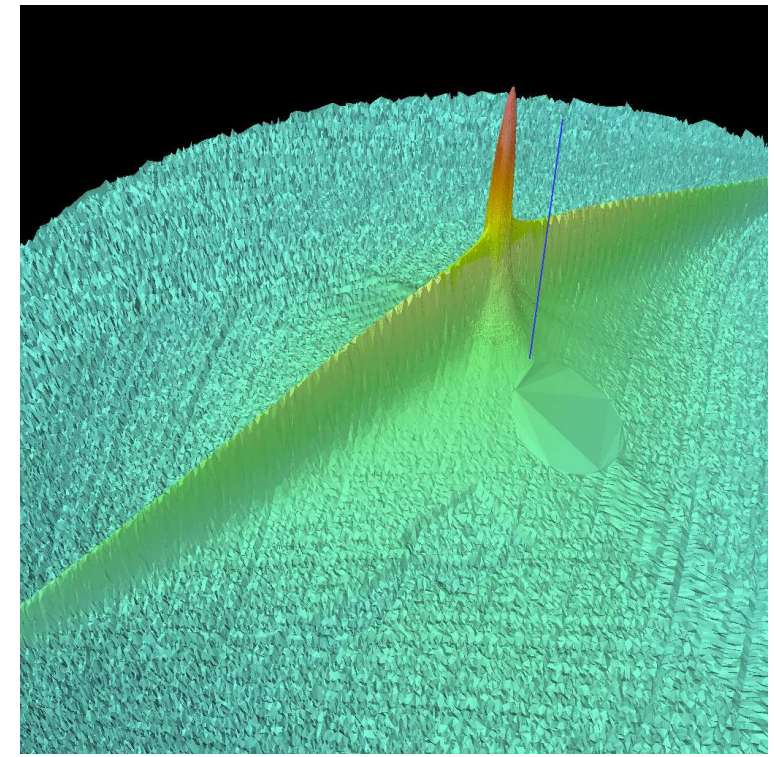

Figure 5. Reflection distribution the same front-side mirror at 8 degrees incident angle as derived from a refined measurement. While the grate in the scatter plane is accounted for now, the gap caused by shadowing from the detector-head is obvious.

\section{ANGULAR RESOLVED BRTD MEASUREMENT RESULTS}

The BRTD of three samples has been measured under the incident angles $\theta_{\text {in }}=5,15,30,45,60$ degrees. These measurements have been completed all all three laboratories participating in the study. The results were shared and the profiles of the BRTD in the scatter plane are compared.

The measured signal depends on the angular response of the detector as well as the solid angle of the source. For finer structures, this results in averaging depending on the both the beam angle of the lamp and the view angle of the detector. This is less obvious for scattering samples, but determines the measurement of highly specular samples with peak shapes approximating delta-functions. Such peaks of ideally infinitely high intensity and infinitely small solid angle lead to singularities result in a valid integral, but the solid angle cannot be resolved infinitely. To allow any comparison of such measurements, the reference beam must thus be considered. It is shown that for the scattering plane, the alignment of the beam was better then 0.25 degrees for any device. The beam angles, defined as the angle where the intensity is more then half of the beam maximum, were found to be below one degree for all three devices. 


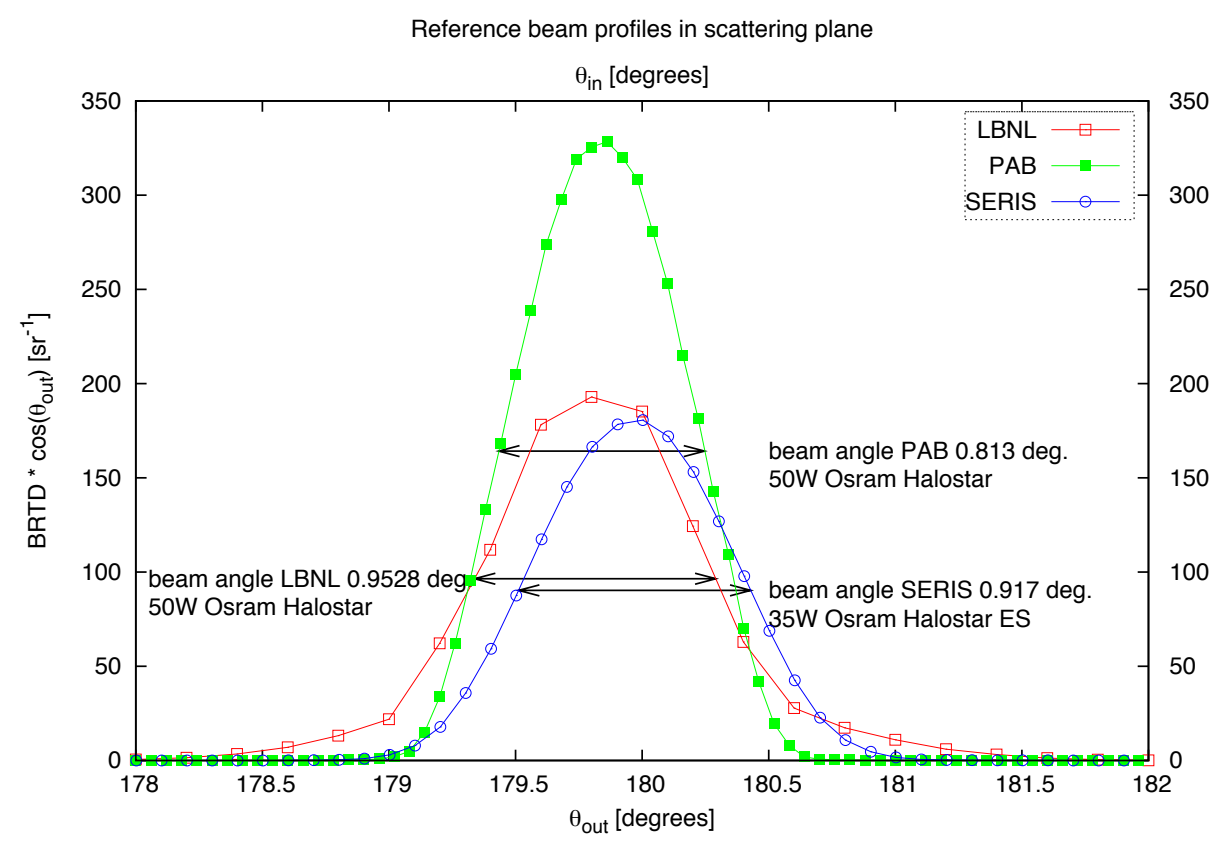

Figure 6. Unobstructed beam profiles. The different beam integral reflect the different lamps used. The beam angle is marked as the angular range where the intensity is above half the maximum beam intensity. 


\section{1 clear low-e glass}

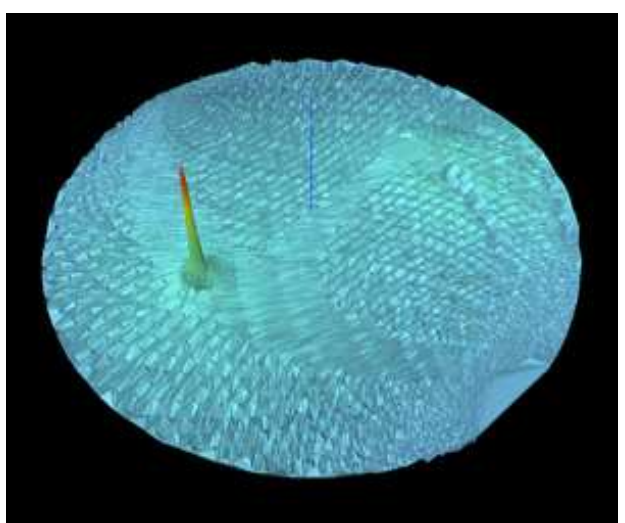

Figure 7. Clear low-e glass: cosine-weighted BRTD measured for incident angle $\theta_{i n}=45$, reflection, logarithmic scale.

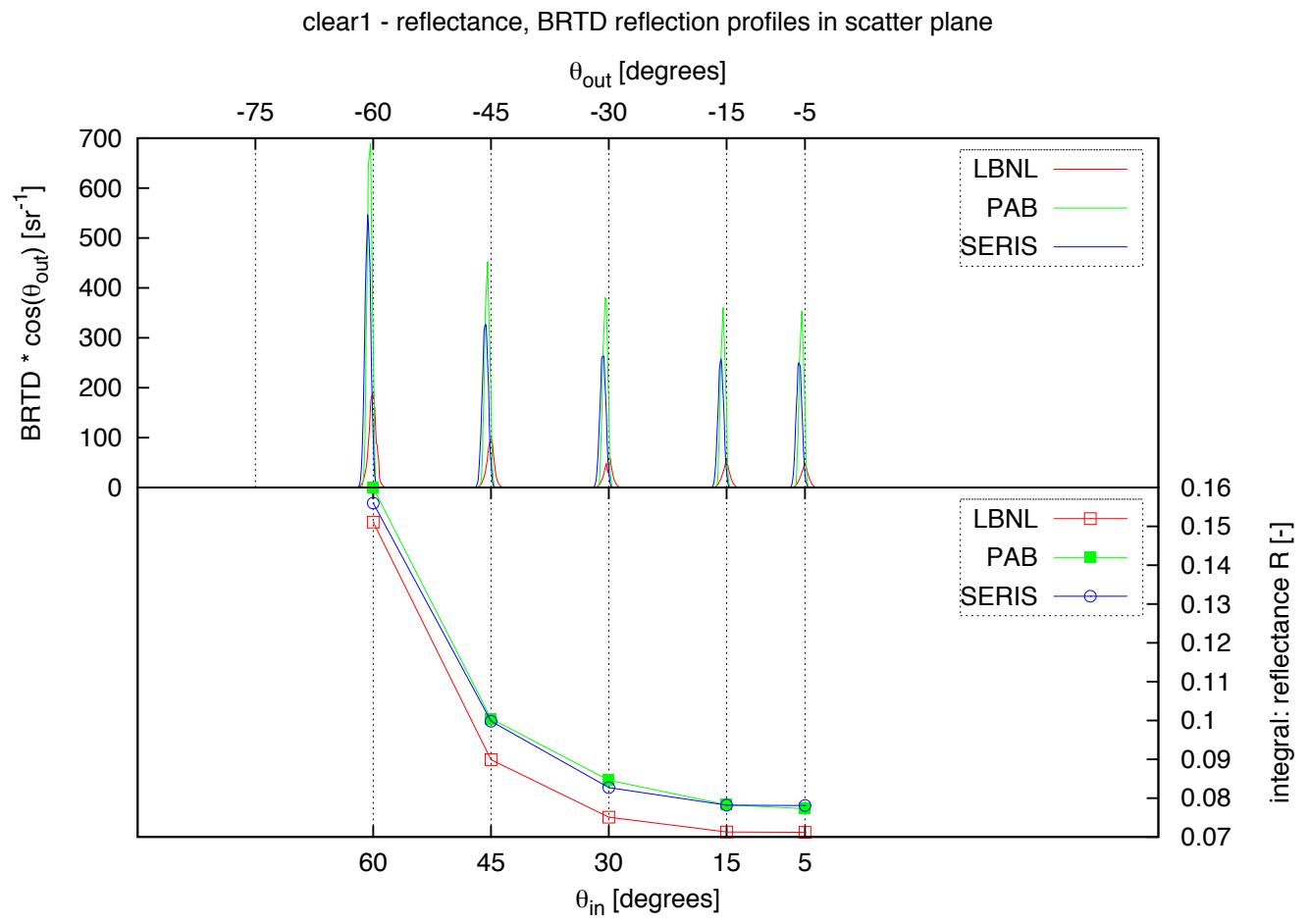

Figure 8. Clear low-e glass: BRTD profiles in the scatter plane for six incident angles of 5, 15, 30, 45 and 60 degrees, reflection. 


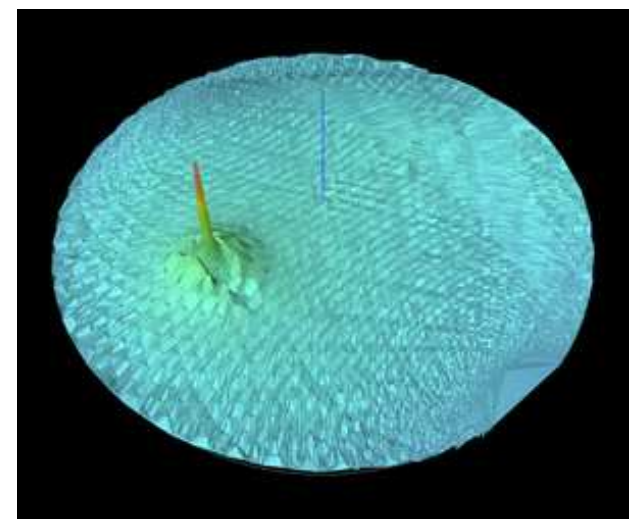

Figure 9. Clear low-e glass: cosine-weighted BRTD measured for incident angle $\theta_{i n}=45$, transmission, logarithmic scale.

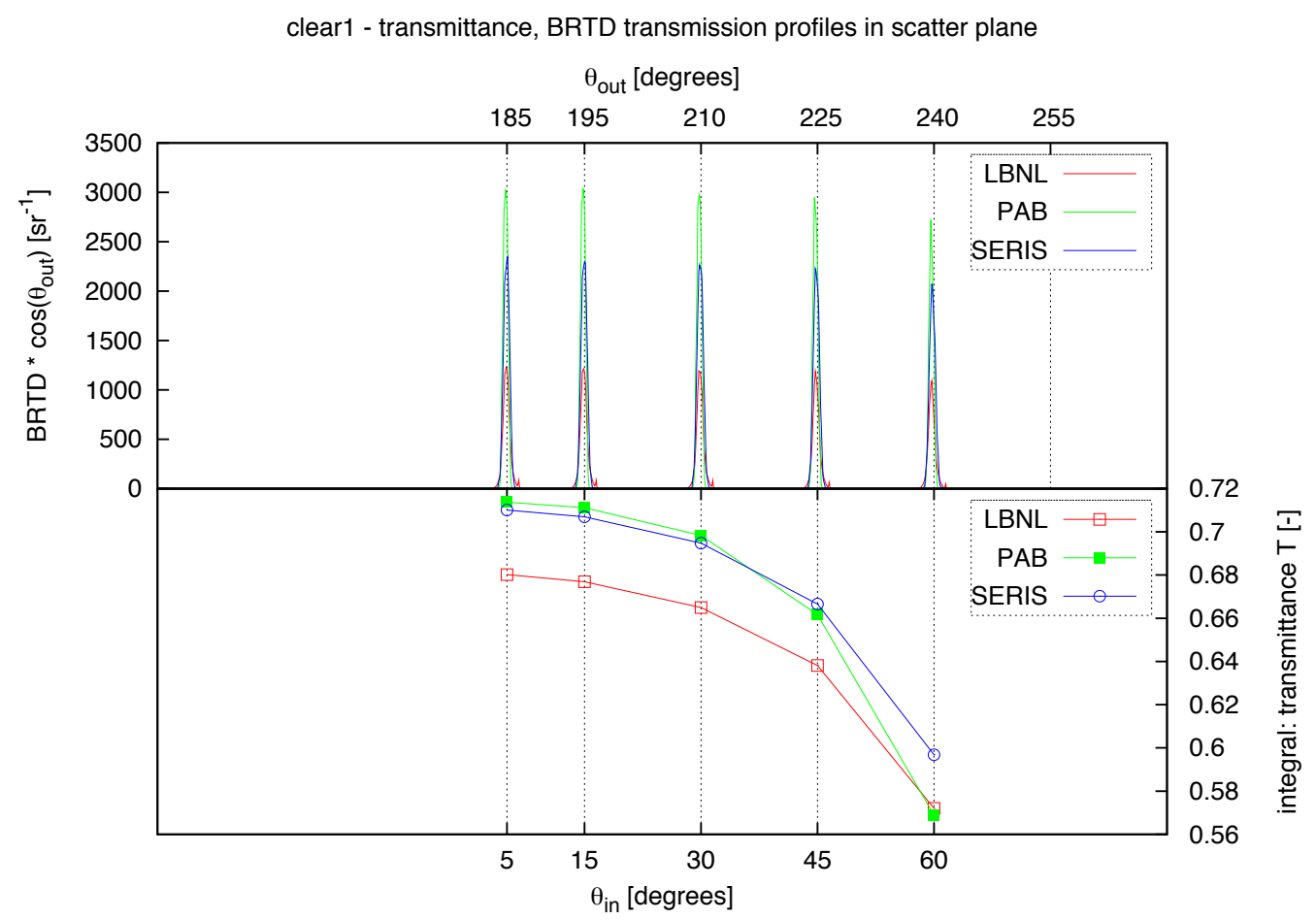

Figure 10. Clear low-e glass: BRTD profiles in the scatter plane for six incident angles of 5, 15, 30, 45 and 60 degrees, transmission. 


\section{2 scattering ground glass}

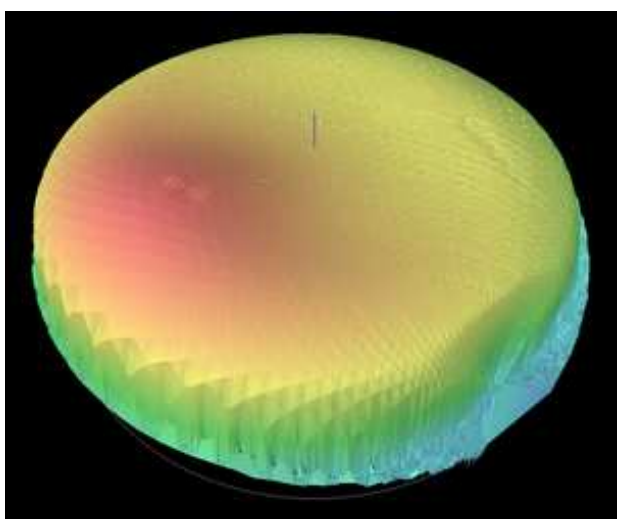

Figure 11. Ground glass: cosine-weighted BRTD measured for incident angle $\theta_{i n}=45$, reflection, logarithmic scale.

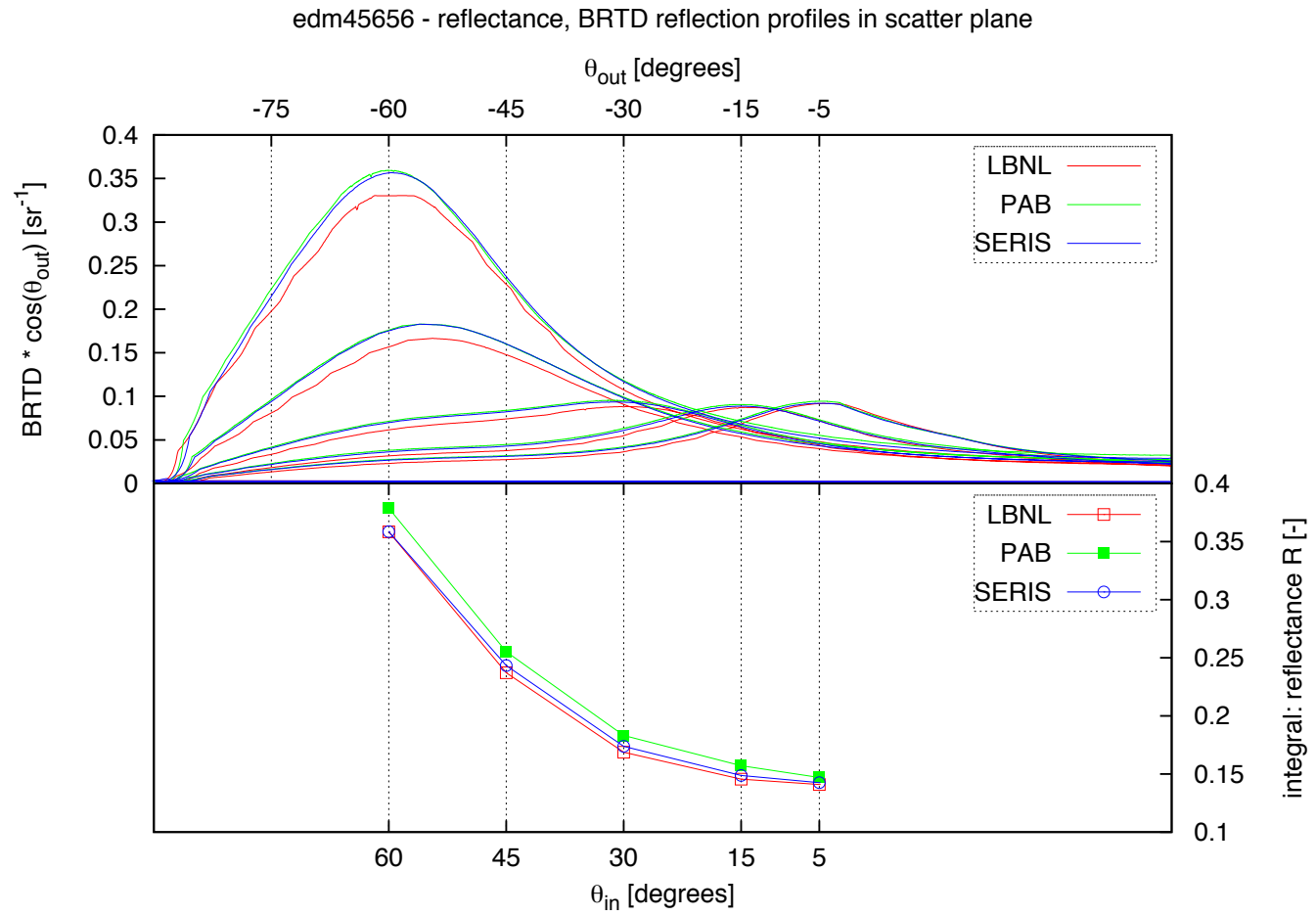

Figure 12. Ground glass: BRTD profiles in the scatter plane for six incident angles of 5, 15, 30, 45 and 60 degrees, reflection. 


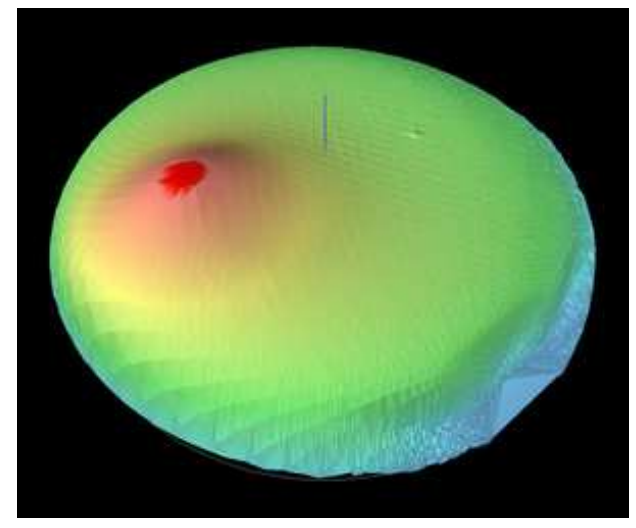

Figure 13. Ground glass: cosine-weighted BRTD measured for incident angle $\theta_{i n}=45$, transmission, logarithmic scale.

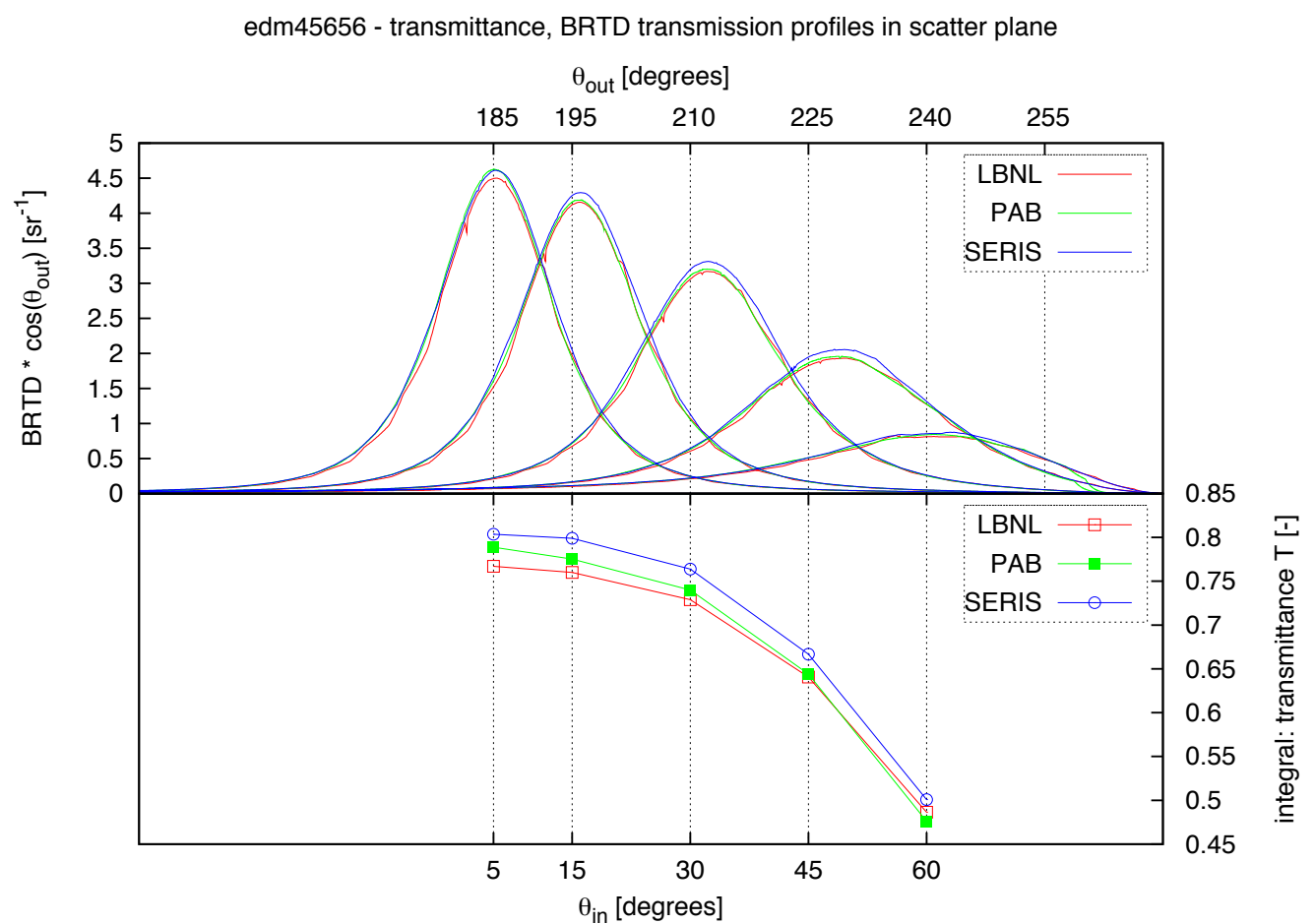

Figure 14. Ground glass: BRTD profiles in the scatter plane for six incident angles of 5, 15, 30, 45 and 60 degrees, transmission. 


\section{3 scattering aluminum front-side mirror}

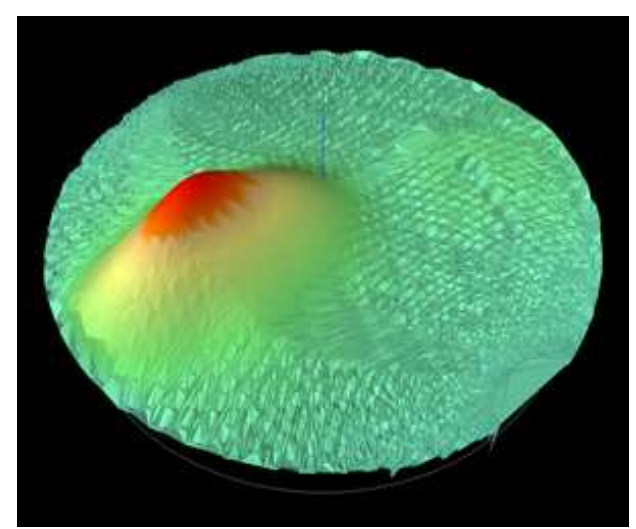

Figure 15. Scattering aluminum front-side mirror: cosine-weighted BRTD measured for incident angle $\theta_{i n}=45$, reflection, logarithmic scale. On the right side, a weak signal is visible due to light reflected from the sample to the wall and back into the reflector.

miro20-2000 - reflectance, BRTD reflection profiles in scatter plane

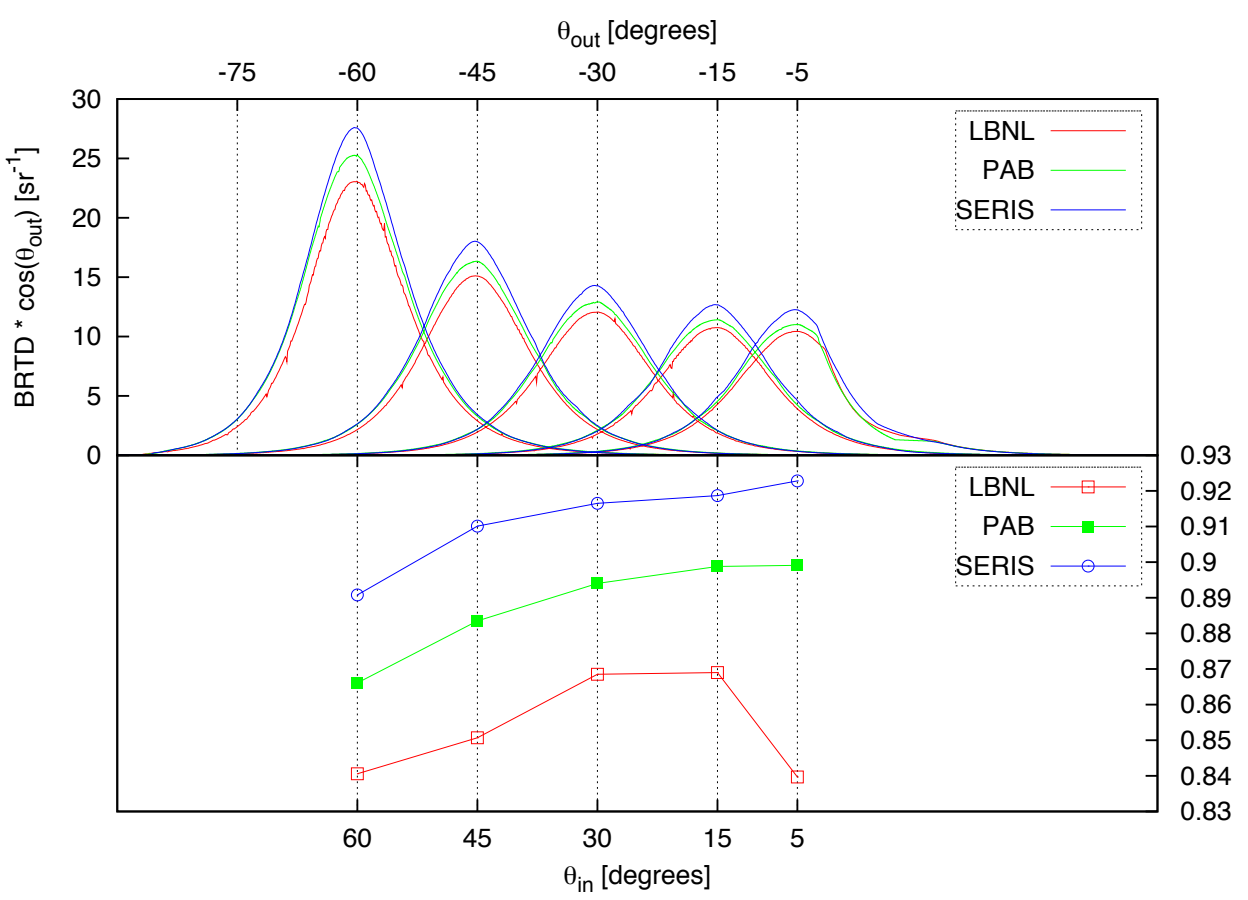

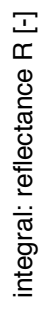

Figure 16. Scattering aluminum front-side mirror: BRTD profiles in the scatter plane for six incident angles of $5,15,30$, 45 and 60 degrees. 


\section{DISCUSSION}

\subsection{Agreement of results, identified sources of error}

Measurements using an integrating sphere are compared to the integrals of the BRTDs measured using two goniophotometers. A detector-filter configuration leading to a $\mathrm{v}(\lambda)$ response curve was chosen to allow a photometric comparison. For transmission, a relative agreement of the two different measurement techniques was achieved of $\pm 2 \%$. Higher relative deviations were observed for reflection, with up to $\pm 16 \%$ for clear glass. Lower deviations were observed for samples with higher reflectance.

Using a different detector-filter configuration, the set of three samples was measured at all three participating laboratories for five incident angles in the scatter plane $\left(\phi_{i n}=0, \theta_{i n}=5,15,30,45,60\right.$ degrees). Again, the integrals of the transmission and reflection measurements were compared. For transmission, a range of $\pm 5 \%$ was observed regardless the incident angle. For reflection, again, less agreement on the results is seen within up to $\pm 12 \%$ for clear glass. Again, the range gets narrower for higher reflectances.

The angular resolved measurements were overlaid only as profiles in the scatter plane as the samples are assumed to be isotropic. For the transmission measurements, the measured peaks show a deviation from the expected specular direction. This may be the result of multiple reflections especially in the clear glass which has to be further studied. Besides the differences in amplitude that are to be expected according to the differences of the BRTDs' integrals, especially the differences in the shape of specular peaks agree with the reference beam angles. For strongly scattering samples, measurement artifacts could be observed that are due to shadowing by the detector head. Some weak signals visible in the logarithmic projections of the full scan indicate that light reflected back from the walls was recorded.

The first potential source of error in the measurement that was identified is the decrease of the beam flux after the initial proposed stabilization time of 30 minutes, which was thus extended to 45 minutes but obviously depends on the lamp type. Even after the extended stabilization time a slow decrease of about $0.06 \%$ per hour can become significant when many sequential measurements are performed over a longer time span. This could be compensated for by applying a time-dependent correction factor, but can be easily avoided by repeated reference beam measurements, which is the recommended procedure. The configuration of the optical bench requires constant consistency checks. The alignment of the beam, choosing a appropriate beam diameter according to the sample and the suppression of stray light are necessary steps to ensure valid results.

One source of error that was only partially studied is the coplanarity of the sample surface with the measurement plane. For the scatter plane, this was confirmed as the reflective peaks match the expected directions. Similar checks are recommended for a second plane at $\phi=90$ degrees.

Weak signals from light being reflected off the surrounding walls were observed. Such signals may become significant when reflective surfaces in the surrounding are hit by light reflected by a sample, and thus not accounted for in the reference measurement. The observation that the integrals of the BRTD measurements were varying stronger for samples of low reflectance may indicate that, especially the measurement environment needs further investigation to explain the deviations.

\subsection{Conclusions}

A configuration has been set up that allows for the comparison between integrating measurements and integrals of angular resolved measurements of BRTD. This was complemented by qualitative comparisons of the distributions in the scatter plane, assuming isotropic samples. This is the foundation for ongoing comparisons that are meant to limit the error range of BRTD measurements and identify potential sources of error.

Even though the three goniophotometers used in the scope of this initial comparison were configured to achieve direct comparability, the fact that the BRTD measurement happens in an open environment, with different room layouts, surface materials and sources of light leakage, has to be considered as well as the use of samples of unknown properties. As such, the rather high deviations especially for reflection on samples of low reflectance are understandable and actually still appear low when compared to early studies ${ }^{9}$ which revealed deviations of more then $100 \%$. As the devices already offer high angular resolution, good agreement in the beam alignment and angles, and stability of the source was determined, potential for further improvement is seen mostly in further controlling the impact of the surrounding. 


\subsection{Ongoing work}

The thickness of samples is well-known to affect the transmission and reflection measurements especially of scattering materials. Edge-losses are to be considered for transmissive samples. These effects depend on the incident angle as well as the sampled surface and beam diameter and may lead to a systematic error. The device is optimized for flat samples with a high ratio of sampled area to thickness, but in extreme cases the application of models and ray tracing may be required or edge losses be minimized by other means. One approach to quantify the effect of sample thickness and edge losses would be comparing to hemispherical-hemispherical measurements on an integrating sphere.

The comparative measurements as they have been presented require the exchange of the sample set in the next step, to finally arrive at three sets of samples that have been measured at each laboratory and thus complete the round robin procedure. This, together with the knowledge on the flux stability and beam alignment, will allow to identify specific error sources at each laboratory. The influence of the surrounding surfaces and sources has to be studied further.

The regular verification by comparing measurements with previous results will allow quality checks on measurements and are to provide an early indicator for changes in the measurement system.

\section{REFERENCES}

[1] Nicodemus, F. E., Richmond, J. C., Hsia, J. J., Ginsberg, I. W., and Limperis, T., "Geometric considerations and nomenclature for reflectance," Monograph 161, National Bureau of Standards (US) (Oct. 1977).

[2] Torrance, K. E. and Sparrow, E. M., "Theory for off-specular reflection from roughened surfacesw," Journal of the Optical Society of America 57, 1105-1114 (1967).

[3] Dana, K., Van-Ginneken, B., Nayar, S., and Koenderink, J., "Reflectance and Texture of Real World Surfaces," ACM Transactions on Graphics (TOG) 18, 1-34 (Jan 1999).

[4] Matusik, W., Pfister, H., Brand, M., and McMillan, L., "A data-driven reflectance model," ACM Transactions on Graphics 22, 759-769 (July 2003).

[5] Havran, V., Neummann, A., Zotti, G., Purgathofer, W., and Seidel, H.-P., "On cross-validation and resampling of brdf data measurements," in [SCCG '05: Proceedings of the 21st Spring Conference on Computer Graphics], 161-168, ACM SIGGRAPH and EUROGRAPHICS, ACM, Budmerice, Slovakia (May 2005).

[6] Apian-Bennewitz, P. and von der Hardt, J., "Enhancing and calibrating a goniophotometer - Measurement and Analysis," Solar Energy Materials and Solar Cells 54, 309-322 (13 July 1998).

[7] Apian-Bennewitz, P., "pab Ltd Gonio-Photometer II." company website (2010).

[8] "TSO 11664-1:2008(E)/CIE S 014-1/E:2006," CIE Colorimetry Part 1 (2008).

[9] Leonard, T. and Pantoliano, M., "BRDF Round Robin," Stray light and contamination in optical systems (Jan. 1989). 


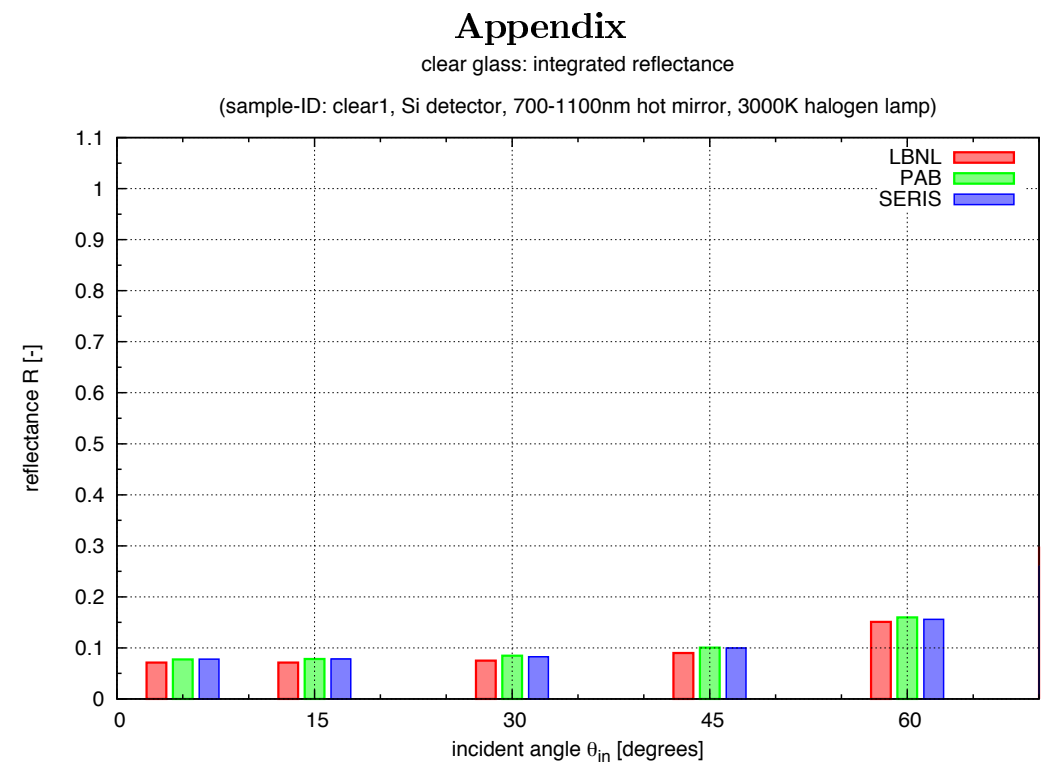

Figure 17. Clear glass: reflectance for six incident angles $\theta_{i n}=5,15,30,45,60,75$.

clear glass: integrated transmittance

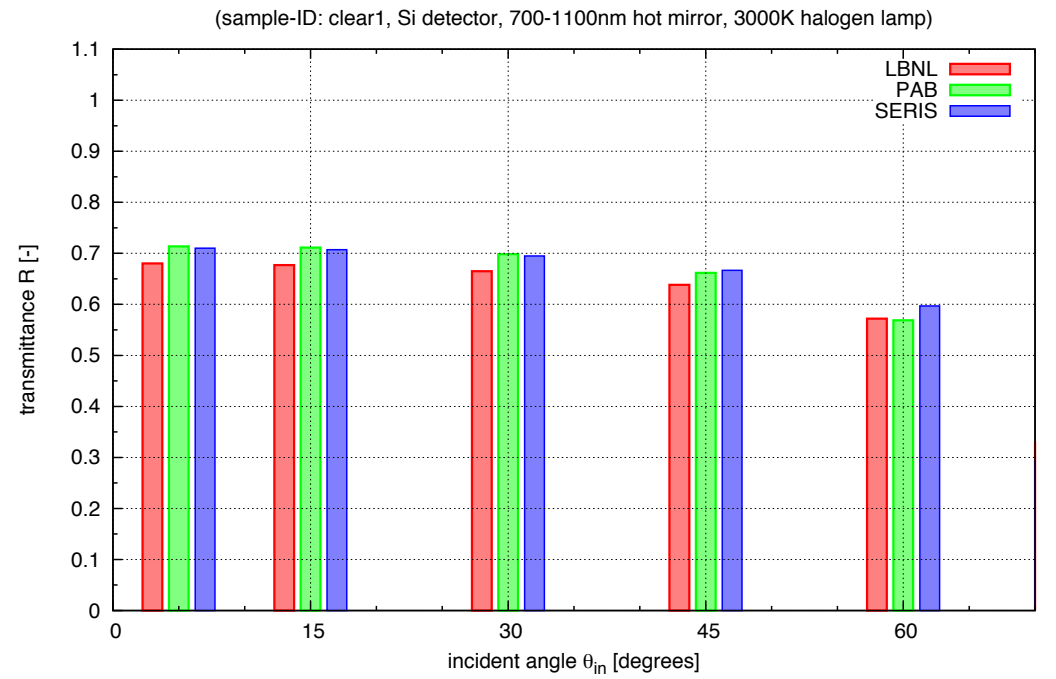

Figure 18. Clear glass: transmittance for six incident angles $\theta_{i n}=5,15,30,45,60,75$.

\begin{tabular}{|c|c|c|c|c|c|c|}
\hline clear1 & \multicolumn{3}{|c|}{ T } & \multicolumn{3}{c|}{ R } \\
\hline$\theta_{\text {in }}$ & LBNL & PAB & SERIS & LBNL & PAB & SERIS \\
\hline 5 & 0.6802 & 0.7137 & 0.7101 & 0.0712 & 0.0773 & 0.0781 \\
\hline 15 & 0.6769 & 0.7112 & 0.7070 & 0.0713 & 0.0783 & 0.0782 \\
\hline 30 & 0.6650 & 0.6983 & 0.6948 & 0.0751 & 0.0846 & 0.0827 \\
\hline 45 & 0.6381 & 0.6617 & 0.6666 & 0.0899 & 0.1005 & 0.0998 \\
\hline 60 & 0.5720 & 0.5689 & 0.5968 & 0.1511 & 0.1599 & 0.1560 \\
\hline
\end{tabular}

Table 6. Clear glass: results from integrated transmission and reflection measurements for sample clear1 using a Si-detector, halogen lamp and a 700-1100nm hot mirror on Goniophotometers at all three labs. 
ground glass: integrated reflectance

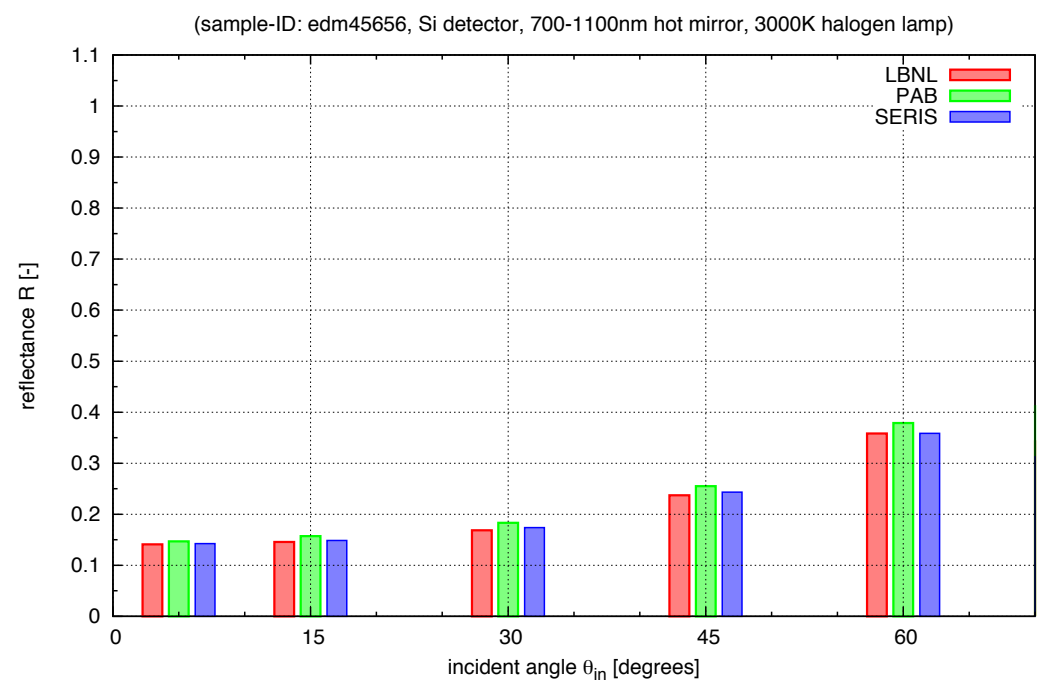

Figure 19. Ground glass: reflectance for six incident angles $\theta_{i n}=5,15,30,45,60,75$.

ground glass: integrated transmittance

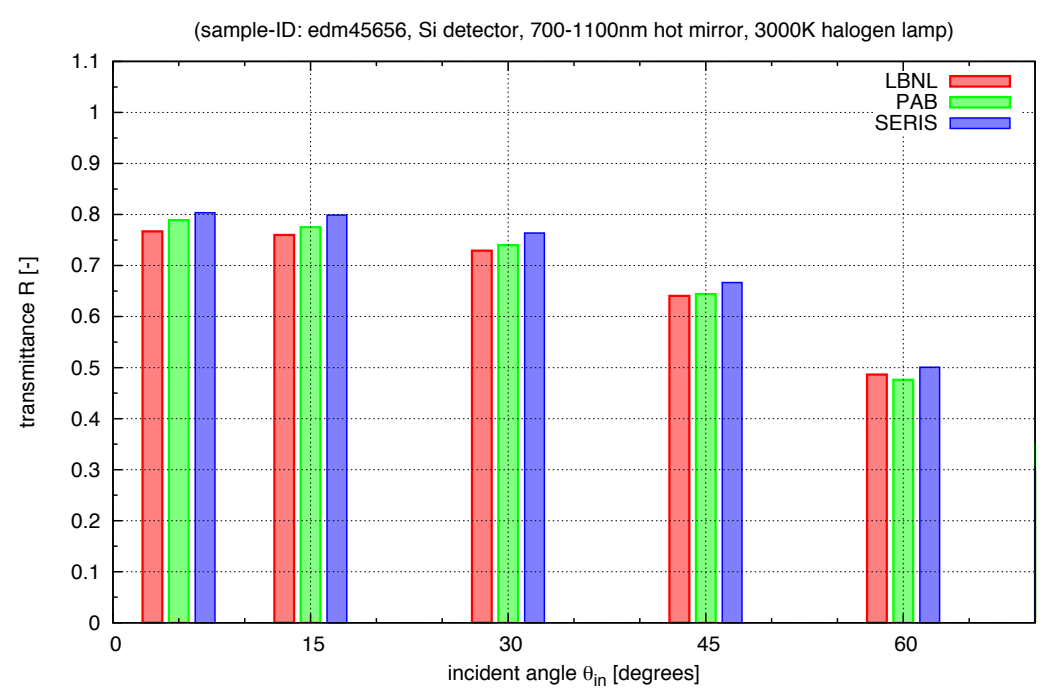

Figure 20. Ground glass: transmittance for six incident angles $\theta_{i n}=5,15,30,45,60,75$.

\begin{tabular}{|c|c|c|c|c|c|c|}
\hline edm45656 & \multicolumn{3}{|c|}{ T } & \multicolumn{3}{c|}{ R } \\
\hline$\theta_{\text {in }}$ & LBNL & PAB & SERIS & LBNL & PAB & SERIS \\
\hline 5 & 0.7670 & 0.7888 & 0.8037 & 0.1410 & 0.1470 & 0.1426 \\
\hline 15 & 0.7600 & 0.7753 & 0.7989 & 0.1456 & 0.1572 & 0.1487 \\
\hline 30 & 0.7290 & 0.7400 & 0.7638 & 0.1687 & 0.1832 & 0.1738 \\
\hline 45 & 0.6406 & 0.6440 & 0.6668 & 0.2372 & 0.2552 & 0.2434 \\
\hline 60 & 0.4865 & 0.4761 & 0.5008 & 0.3584 & 0.3787 & 0.3586 \\
\hline
\end{tabular}

Table 7. Ground glass: results from integrated transmission and reflection measurements for sample edm45656 using a Si-detector, halogen lamp and a 700-1100nm hot mirror on Goniophotometers at all three labs. 


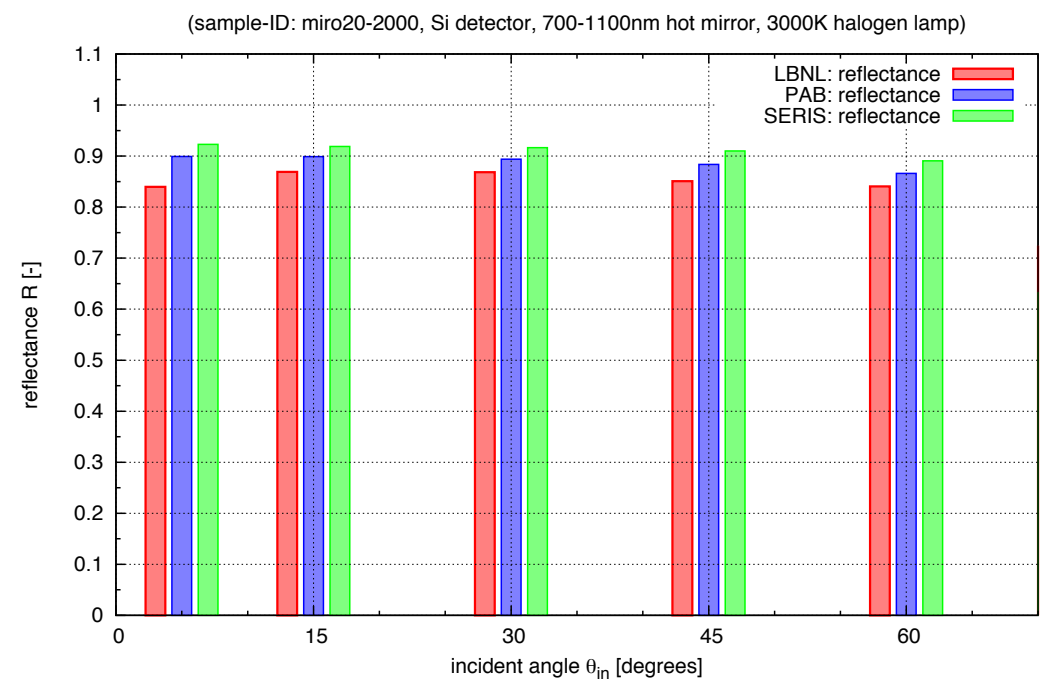

Figure 21. Scattering aluminum mirror: reflectance for six incident angles $\theta_{i n}=5,15,30,45,60,75$.

\begin{tabular}{|c|c|c|c|}
\hline miro20-2000 & \multicolumn{3}{|c|}{$\mathrm{R}$} \\
\hline$\theta_{\text {in }}$ & LBNL & PAB & SERIS \\
\hline 5 & 0.8397 & 0.8991 & 0.9228 \\
\hline 15 & 0.8690 & 0.8988 & 0.9187 \\
\hline 30 & 0.8685 & 0.8940 & 0.9165 \\
\hline 45 & 0.8507 & 0.8835 & 0.9101 \\
\hline 60 & 0.8406 & 0.8661 & 0.8908 \\
\hline
\end{tabular}

Table 8. Scattering aluminum front side mirror: results from integrated reflection measurements for sample miro20-2000 using a Si-detector, halogen lamp and a 700-1100nm hot mirror on Goniophotometers at all three labs. 